\title{
ON THE USE OF COMPRESSIVE SAMPLING FOR WIDE-BAND SPECTRUM SENSING
}

\author{
Dennis Sundman, Saikat Chatterjee, Mikael Skoglund \\ School of Electrical Engineering and ACCESS Linnaeus Centre \\ KTH - Royal Institute of Technology, SE-10044 Stockholm, Sweden \\ denniss@kth.se, sach@kth.se, skoglund@kth.se
}

\begin{abstract}
In a scenario where a cognitive radio unit wishes to transmit, it needs to know over which frequency bands it can operate. It can obtain this knowledge by estimating the power spectral density from a Nyquistrate sampled signal. For wide-band signals sampling at the Nyquist rate is a major challenge and may be unfeasible. In this paper we accurately detect spectrum holes in sub-Nyquist frequencies without assuming wide sense stationarity in the compressed sampled signal. A novel extension to further reduce the sub-Nyquist samples is then presented by introducing a memory based compressed sensing that relies on the spectrum to be slowly varying.
\end{abstract}

Index Terms - Compressive sampling, Cognitive radio, power spectrum estimation, sub-Nyquist sampling.

\section{INTRODUCTION}

Compressive Sampling (CS) [1] has attracted much attention in the current literature due to its promise of realizing sub-Nyquist sampling. A signal can be reconstructed from samples obtained at sampling rate lower than the Nyquist rate if the signal is sparse in some domain. Recently CS $[2,3]$ has been employed in wide-band spectrum sensing in Cognitive Radio (CR) [4] applications because the spectrum has an underlying sparsity. In practice, at a given time, the full wide-band spectrum is inherently sparse in the sense that only a few users occupy some of many available frequency bands.

In a CR scenario we are interested in sensing wide-band signals in order to detect vacant frequency bands. A straightforward approach to detect the unoccupied frequency bands can be based on evaluating the Power Spectral Density (PSD) from Nyquist rate samples. However, wide-band signal sampling at Nyquist rate is a major challenge.

An autocorrelation based method of estimating the PSD from CS samples has been proposed in [3, 5]. The connection between an analog signal and the CS samples is established through using an analog-to-information converter (AIC) [6], [7]. An AIC takes an analog signal as input and gives the CS samples as output.

In the standard CS set-up, CS samples and Nyquist rate samples are connected via a linear transformation where the sensing matrix is random in nature [8].

In this paper we deal with the practical problem of estimating the autocorrelation coefficients of a signal from CS samples. The PSD can then be obtained as the Fourier transform of the autocorrelation coefficients. Due to the random sensing matrix, the signal obtained from the CS samples is in general not wide sense stationary (WSS). We note that stationarity was assumed in [3], although

This work was funded in part by VINNOVA. autocorrelation coefficients for non-WSS signals have no physical meaning.

We follow the guide-lines of [3] and notice that it is possible to sample in a significantly lower rate than the Nyquist rate in the task of reconstructing the PSD (with a for detection purposes acceptable degree of accuracy). In our approach, instead of estimating the autocorrelation coefficients from a CS sample vector (i.e. a vector consisting of successive CS samples), a correlation matrix is estimated from several CS sample vectors. Effectively, this strategy may lead to acquiring more total samples than the Nyquist rate samples. However, we no longer need the assumption of WSS and although the total number of samples is increased the actual sampling rate can be reduced significantly.

We further present a novel extension of the CS PSD estimation by assuming that the spectrum is slowly time-varying in the sense that the change in spectrum between two neighboring time-instants is small. This assumption is motivated by the fact that there are static components in the frequency spectrum; broadcast TV and radio, mobile traffic, wireless Internet traffic, etc.. According to this assumption, we notice that the difference between two spectrum realizations taken close in time is likely to be sparser than each spectrum by itself. We propose to benefit from this increased sparsity by taking fewer number of samples while still reconstructing the current spectrum without loss of accuracy. We are able to do this by introducing a memory in the CS process. Since we are only interested in knowing which frequency bands are free or occupied we realize detection by thresholding.

This paper is organized as follows. We discuss CS and how it can be applied for wide-band spectrum estimation in section 2 . In section 3 we discuss the issue of detection from a spectrum estimation and we present our alternative method which takes into account the aforementioned memory of the CS process. The performance of the proposed method is verified through simulations in section 4 . Section 5 concludes this paper.

\section{CS BASED WIDE-BAND SPECTRUM SENSING}

This section starts with a short definition of notation. We follow with a subsection describing the general CS method and then a description how this can be used for wide band spectrum sensing.

Italic letters, i.e. $M$, will refer to constants. Bold face capital letters, i.e. $\boldsymbol{\Phi}$, denotes matrices and bold face lower-case letters $\mathbf{x}$ denotes vectors. The notation * denotes, in case of a scalar the complex conjugate and in case of a matrix the Hermitian operator. The $p$-norm is defined as $\|\mathbf{x}\|_{p}=\left(\sum_{\forall i}\left|x_{i}\right|^{p}\right)^{1 / p}$. The $(i, j)$ 'th element of a matrix $\boldsymbol{\Phi}$ is denoted by $\{\boldsymbol{\Phi}\}_{i, j}$. 


\subsection{Compressed Sensing}

In the Compressed Sensing (CS) framework one assumes that an $N$ dimensional signal $\mathbf{s}$ consists of samples presented at the Nyquist rate. The signal is sparse in a transform domain. Let us denote the $N \times N$ transform matrix $\boldsymbol{\Psi}$, in which the signal is sparse. A $K$ sparse signal has at most $K$ non-zero components. In other words, if $\mathbf{s}$ is $K$-sparse in the transform domain $\Psi$, then $\|\Psi \mathbf{s}\|_{0} \leq K$, where $\|\cdot\|_{0}$ refers to the number of non-zero coefficients.

To obtain the CS samples from an analog signal a hardware device commonly known as AIC [6] is used. Assume that sampling at the Nyquist rate yields $N$ samples. The purpose of the AIC is then to collect $M$ samples such that $M<N$ according to the CS procedure, from which a Nyquist sampled signal then can be reconstructed using CS reconstruction. Several AIC architectures have been proposed in the literature, e.g. random demodulator [9], random filtering and random convolution $[10,11,12]$. In particular, the random demodulator has shown promising results in acquiring CS samples at a sampling rate exponentially lower than the Nyquist-rate.

A common interpretation of an AIC is as sampling done at Nyquist-rate followed by an $M \times N$ (down-)sampling matrix, $\boldsymbol{\Phi}$. For reconstruction to be possible, $M$ has to be sufficiently large. According to E. Candés and T. Tao if $M$ is chosen as

$$
M \gtrsim K \log _{10} N,
$$

the signal can be recovered with high probability if the elements in $\boldsymbol{\Phi}$ are selected independently at random [13] (also see [8, 14]). A matrix $\boldsymbol{\Phi}$, with its elements chosen in this way (and satisfying the aforementioned inequality of $M$ ), is said to obey the Restricted Isometric Property (RIP), which is necessary for reconstruction of

$$
\mathbf{y}=\mathbf{\Phi} \mathbf{s}
$$

The reconstruction procedure takes the form of a convex problem:

$$
\hat{\mathbf{s}}=\arg \min _{\mathbf{s}}\|\Psi \mathbf{s}\|_{1} \quad \text { s.t. } \quad \mathbf{y}=\mathbf{\Phi} \hat{\mathbf{s}},
$$

or equivalently,

$$
\hat{\mathbf{z}}=\arg \min _{\mathbf{z}}\|\mathbf{z}\|_{1} \quad \text { s.t. } \quad \mathbf{y}=\Psi \boldsymbol{\Phi}^{-1} \hat{\mathbf{z}},
$$

where $\mathbf{z}$ is $K$-sparse. For practical convenience the latter form is used in this paper. The convex problem can be solved by different linear programming techniques, e.g. basis pursuit [15] or iterative greedy algorithms [16]. All convex problems in this paper are solved using the cvx toolkit [17].

\subsection{Wide band spectrum Sensing}

The CS technique relies on sparsity; the sparser signal the more efficient is the method. It is realized that the edge spectrum $\mathbf{z}_{x}$ (derivative of the PSD) is sparser than the spectrum alone. The PSD is obtained from the autocorrelation coefficients via the Fourier transform. Denoting the edge spectrum by $\mathbf{z}_{x}$ we can form the relation between the edge spectrum and the autocorrelation coefficients, analogously to [2], as

$$
\mathbf{r}_{x}=(\Gamma \mathcal{W} \mathcal{F})^{-1} \mathbf{z}_{x},
$$

where $\mathcal{W}$ is a $2 N \times 2 N$ wavelet smoothing matrix, $\mathcal{F}$ is the $2 N \times 2 N$ Discrete Fourier Transform (DFT) matrix and $\Gamma$ is the approximated, by first-order difference, derivative implemented as the $2 N \times 2 N$ matrix

$$
\Gamma=\left[\begin{array}{ccccc}
1 & 0 & 0 & \cdots & 0 \\
-1 & 1 & 0 & \cdots & 0 \\
0 & \ddots & \ddots & \ddots & \vdots \\
\vdots & \ddots & -1 & 1 & 0 \\
0 & \cdots & 0 & -1 & 1
\end{array}\right]
$$

For simplicity we will denote $\mathbf{G}=(\Gamma \mathcal{W} \mathcal{F})^{-1}$.

The spectrum sensing scheme presented in [2] is based on full ADC sampling i.e. according to the Nyquist rate. This approach is extended in [3] to the case where we only have access to the CSsampled signal

$$
\mathbf{y}=\mathbf{\Phi} \mathbf{x}
$$

We are now left with the task of finding a linear transform that takes us from the CS-sampled signal to the autocorrelation coefficients $\mathbf{r}_{x}$. A linear relation, based on the correlation matrix relation $\mathbf{R}_{y}=\boldsymbol{\Phi} \mathbf{R}_{x} \boldsymbol{\Phi}^{*}$ is found

$$
\mathbf{r}_{y}^{\prime}=\boldsymbol{\Phi}_{\mathbf{I I}} \mathbf{r}_{x}
$$

where $\mathbf{r}_{y}^{\prime}$ is defined as the first row (reversed) concatenated with the first column (without the first component)

$$
\mathbf{r}_{y}^{\prime} \triangleq\left[0\left\{\mathbf{R}_{y}\right\}_{1, N} \cdots\left\{\mathbf{R}_{y}\right\}_{1,1}\left\{\mathbf{R}_{y}\right\}_{2,1} \cdots\left\{\mathbf{R}_{y}\right\}_{N, 2}\right]^{T}
$$

and $\mathbf{r}_{x}$ is defined as

$$
\mathbf{r}_{x} \triangleq\left[0 r_{x}(-N+1) \cdots r_{x}(0) \cdots r_{x}(N-1)\right]^{T}
$$

where the zeros are added for implementation convenience. The vector $\mathbf{r}_{y}^{\prime}$ is defined from the correlation matrix rather than the autocorrelation coefficients because $\boldsymbol{\Phi}$ does, in general, not preserve WSS in the measurements.

Remark 1 The linear sensing operation (7), can be realized as filtering with the filter described by the matrix $\mathbf{\Phi}$. This filter corresponds to a time-varying filter. It is well known [18] that timevarying filters do not preserve the WSS property in signals.

The main difference between the approach in [3] and the one presented here lies in the definition of $\mathbf{r}_{y}^{\prime}$. The relation matrix $\boldsymbol{\Phi}_{\mathbf{I I}}$ is the same as in [3] and is defined as

$$
\boldsymbol{\Phi}_{\mathbf{I I}}=\left[\begin{array}{ll}
\overline{\boldsymbol{\Phi}} \boldsymbol{\Phi}_{1} & \overline{\boldsymbol{\Phi}} \boldsymbol{\Phi}_{2} \\
\mathbf{\Phi} \boldsymbol{\Phi}_{3} & \mathbf{\Phi} \boldsymbol{\Phi}_{4}
\end{array}\right]
$$

where we denote the $(i, j)$ 'th element of $\boldsymbol{\Phi}$ by $\phi_{i, j}$, then the $M \times N$ matrix $\overline{\boldsymbol{\Phi}}$ has its $(i, j)$ 'th element given by

$$
\{\overline{\mathbf{\Phi}}\}_{i, j}=\left\{\begin{array}{cc}
0 & i=1, j=1, \ldots, N \\
\phi_{M+2-i, j}^{*} & i \neq 1, j=1, \ldots, N
\end{array}\right.
$$

The $N \times N$ matrices $\boldsymbol{\Phi}_{1}, \boldsymbol{\Phi}_{2}, \boldsymbol{\Phi}_{3}$, and $\boldsymbol{\Phi}_{4}$ are

$$
\begin{aligned}
& \boldsymbol{\Phi}_{1}=\operatorname{hankel}\left(\left[\mathbf{0}_{N \times 1}\right],\left[0 \phi_{1,1} \cdots \phi_{1, N-1}\right]\right), \\
& \boldsymbol{\Phi}_{2}=\operatorname{hankel}\left(\left[\phi_{1,1} \cdots \phi_{1, N}\right],\left[\phi_{1, N} \mathbf{0}_{1 \times(N-1)}\right]\right) \text {, } \\
& \boldsymbol{\Phi}_{3}=\operatorname{toeplitz}\left(\left[\mathbf{0}_{N \times 1}\right],\left[0 \phi_{1, N}^{*} \cdots \phi_{1,2}^{*}\right]\right) \text {, } \\
& \boldsymbol{\Phi}_{4}=\operatorname{toeplitz}\left(\left[\phi_{1,1}^{*} \cdots \phi_{1, N}^{*}\right],\left[\phi_{1,1}^{*} \mathbf{0}_{1 \times(N-1)}\right]\right) \text {. }
\end{aligned}
$$


Here hankel(c, r) denotes a Hankel matrix (i.e., symmetric and constant across the anti-diagonals) whose first column is $\mathbf{c}$ and whose last row is $\mathbf{r}$, toeplitz $(\mathbf{c}, \mathbf{r})$ denotes a Toeplitz matrix (i.e., symmetric and constant across the diagonals) whose first column is $\mathbf{c}$ and whose first row is $\mathbf{r}$. The vector $\mathbf{0}_{N \times 1}$ is a column of $N$ zeros and $\mathbf{0}_{1 \times N-1}$ is a row of $N-1$ zeros.

A straight-forward method to estimate $\mathbf{r}_{y}^{\prime}$ is to first estimate $\mathbf{R}_{y}$ and then pick the corresponding elements from the matrix as in (9). We now have linear relations between the CS-sampled signal and the edge spectrum. The convex problem of estimating the edge spectrum becomes

$$
\hat{\mathbf{z}}_{x}=\arg \min _{\mathbf{z}}\|\mathbf{z}\|_{1} \quad \text { s.t. } \quad \mathbf{r}_{y}^{\prime}=\boldsymbol{\Phi}_{\mathbf{I I}} \mathbf{G} \hat{\mathbf{z}}_{x} .
$$

Finally, the estimate of the PSD can be achieved from the edge spectrum as [2]

$$
S(n)=\sum_{k=1}^{n} \hat{z}(k) \quad n=1, \ldots, 2 N-1 .
$$

\section{SPECTRAL DETECTION WITH AND WITHOUT MEMORY}

In this section we investigate how to use the estimate of the PSD in (14) in order to determine which frequency bands are available for CR transmission. First we formulate our detection problem [18] and introduce a method for setting the threshold, then we introduce the memory.

\subsection{Wide band spectral detection based on the PSD}

Having access to the estimate (14) we consider how to use this information to determine which bands in $S(n)$ that are free for transmission. Firstly, we define the wide-band PSD estimate vector

$$
\begin{aligned}
\mathbf{s} & =\left[\begin{array}{llll}
S(1) & S(2) & \cdots & S(2 N-1)
\end{array}\right]^{T} \\
& =\left[\begin{array}{llll}
\mathbf{s}_{1}^{T} & \mathbf{s}_{2}^{T} & \cdots & \mathbf{s}_{B}^{T}
\end{array}\right]^{T},
\end{aligned}
$$

where $\mathbf{s}_{i}$ is a vector representing the PSD over the known frequency sub-band, $i$, and $B$ is the total number of sub-bands. In practice the number of components in each vector $\mathbf{s}_{i}$ does not need to be the same. Now we define the band-vector $\mathbf{b}$ as

$$
\mathbf{b}=\left[\begin{array}{llll}
b_{1} & b_{2} & \cdots & b_{B}
\end{array}\right]
$$

where $b_{i}=\sum_{\forall j} s_{i}(j)$ is the sum over all components in each frequency band and can be thought of as the total energy in that band. In order to find the occupied bands in $\mathbf{b}$, we determine a threshold value $\gamma$, above which the band is considered occupied and below which it is considered vacant. We sort $\mathbf{b}$ in descending order and call the sorted vector $\mathbf{b}_{\mathrm{s}}$. Then take the difference between each consecutive element in $\mathbf{b}_{\mathrm{s}}$ and locate the maximum value

$$
\max \left[\begin{array}{llll}
b_{\mathrm{s}, 1}-b_{\mathrm{s}, 2} & b_{\mathrm{s}, 2}-b_{\mathrm{s}, 3} & \cdots & b_{\mathrm{s}, B-1}-b_{\mathrm{s}, B}
\end{array}\right]
$$

Let the maximum value be $b_{\mathrm{s}, i-1}-b_{\mathrm{s}, i}$, then $b_{\mathrm{s}, i-1}$ is chosen as the threshold value $\gamma$. A requirement for the threshold value to be meaningful is that at least one band has to be occupied. It is also required that the total energy from the weakest occupied band contains "much" more energy compared to a vacant frequency band than it does to all the other occupied bands. as

We are now ready to write the frequency band detection problem

$$
b_{k} \underset{\mathcal{H}_{0}}{\stackrel{\mathcal{H}_{1}}{\gtrless}} \gamma \quad k=1,2, \ldots, B .
$$

Where $\mathcal{H}_{1}$ and $\mathcal{H}_{0}$ denote the hypothesis of a primary signal being present and absent, respectively. The choice of $\gamma$ does not effect the ability of the system to perform at sub-Nyquist rates, even in the memory-based scenario described later. It threshold value only effects the probability of miss or false alarm in the sub-bands of the current PSD.

\subsection{No Memory}

We now introduce our proposed method of estimation of PSD. Assume an AIC that satisfies the RIP (1) gives $M$ measurements. We estimate the correlation matrix of $\mathbf{y}$, as

$$
\hat{\mathbf{R}}_{y}=\frac{1}{L} \sum_{l=1}^{L} \mathbf{y}_{l} \mathbf{y}_{l}^{*},
$$

where $L$ needs to be sufficiently large to achieve a good quality estimate. We obtain an estimate of the vector $\hat{\mathbf{r}}_{y}^{\prime}$ according to (9), and solve the convex problem (13).

If we require $L$ samples for the estimation of $\hat{\mathbf{R}}_{y}$ and $\mathbf{y}$ has the size $M$, this procedure requires a total number of $L M$ samples for each realization of the power spectrum. The total number of samples $L M$ may not be smaller than a conventional sampling of $N$ samples, but these samples can be picked at an (exponentially [9]) lower sampling rate, according to the aforementioned demodulator.

\subsection{Memory}

In practical CR implementations it is of great interest to reduce the total number of samples $L M$ required in estimation since acquiring each sample has an associated cost in terms of energy.

The method proposed in section 3.2 relies on the accuracy in estimating $\hat{\mathbf{R}}_{y}$. We see in (15) that reducing $L$ sacrifices precision in the estimation process. Therefore we limit ourselves to reducing $M$ which is the number of CS samples in each CS vector $\mathbf{y}$.

We propose to use a memory-based spectrum estimation method that exploits the fact that the PSD is likely to be slow-varying nature. The slow-varying nature comes from the fact that there are many static transmissions in our surroundings. Examples of transmissions that clearly can be considered static are broadcast TV and radio. Moreover, in $\mathrm{CR}$ environments where the freed bands only need to be determined every second, mobile phone calls and wireless Internet can also be considered as static.

Let $C$ be a value describing the changes in the $K$-sparse edge spectrum from one time instance to the following as follows. Assume that $\mathbf{z}_{1}$ is $K$-sparse, the next instance edge spectrum $\mathbf{z}_{2}$ is also $K$-sparse and $\mathbf{z}_{2}-\mathbf{z}_{1}$ is $K_{2}$ sparse, then $C=K_{2} / K$. By this definition, $C$ can take any value between 0 and 2 . For the method to be useful it is necessary that $C<1$. From (1) we then notice that the number of CS samples $M_{2}$ when making use of the memory can, for the $C K$-sparse signal, be chosen as $C M_{1}$. We call this method a memory based compressed sensing. The convex problem at hand then becomes

$$
\hat{\mathbf{z}}_{x}=\arg \min _{\mathbf{z}}\left\|\mathbf{z}-\hat{\mathbf{z}}_{\mathrm{old}}\right\|_{1} \quad \text { s.t. } \quad \mathbf{r}_{y}^{\prime}=\mathbf{\Phi}_{\mathbf{I I}} \mathbf{G} \hat{\mathbf{z}}_{x},
$$


By assuming the change is no more than a specified $C$ (which we assume $<1$ ), we are able to repeat this memory based algorithm consecutively a number of times. Full CS sampling without memory requires $M_{1}$ samples per estimate. In contrast, the improved estimation method based on the system memory requires only $M_{2}=C M_{1}$ samples per estimate. Repeat this algorithm, taking consecutive CS samples $M_{2}$ based on the memory a number of times. The drawback is that if an error occurs, it is likely to remain in the memory until we take a full CS sampling at $M_{1}$ again. By applying the memory CS sampling a number of $Q$ times, we then need to take a total of $L M+L M Q C=L M(1+Q C)$ numbers of samples. If $C<1$ then the number of samples required is smaller with the memory than the method without memory which has a total of $L M+L M Q=L M(1+Q)$ number of samples. We quantify the reduction in frequency by comparing the number of required $C S$ measurements for the memory based algorithm with memory $Q$ to the non-memory case. For this purpose we define the average reduction fraction as

$$
R_{f}=\frac{L\left(M_{1}+Q M_{2}\right)}{L\left(M_{1}+Q M_{1}\right)}=\left\{M_{2}=C M_{1}\right\}=\frac{1+Q C}{1+Q} .
$$

This fraction will become smaller, hence better, as $Q$ increases.

How to choose $C$ depends on how small changes the CR user wants to track. It seems reasonable that the $\mathrm{CR}$ user wants to track quite small changes in the spectrum, i.e. choosing $C$ small, which in comparison to the standard method gives a big improvement to the proposed algorithm.

On the contrary, unless there are frequently significant changes in the spectrum, most likely due to big changes in the surroundings, the memory $Q$ can also be chosen large, which further reduces the number of total samples.

\section{SIMULATIONS}

\subsection{Signal Modeling}

A challenge in simulating the proposed algorithm is obtaining realistic data to be sampled by the AIC. We are interested in the power spectrum which means that the signal needs to be WSS. One can also assume that in practice the signal can be modeled as ergodic. These properties of the signal will however not remain after the sampling.

As mentioned earlier, the AIC can be thought of as a Nyquist sampling followed by the application of a measurement matrix $\mathbf{\Phi}$. Since we do not have access to a real time-continuous signal, we instead construct a discrete signal $\mathbf{x}$ and apply $\mathbf{\Phi}$ directly to that.

To form a signal with the given PSD, we first form a 80-tap AR-filter designed from the Yule-walker equations [19]. By filtering white noise through this filter, we obtain a signal $\mathbf{x}$ with the desired properties. The power spectrum of this signal will look like the top picture in Fig. 3. The oscillating effect in the figure is due to Gibbs phenomenon and can be reduced with larger number of taps in the filter.

\subsection{Parameters and Simulation set-up}

In the following simulations we have set the fraction $C$ of change for the power spectrum to $C=1 / 3$ (see Section 3.3). The value $C=1 / 3$ is chosen because it is easy to implement in this particular realization. As an example of wide-band spectrum our range of interest is $0-5 \mathrm{GHz}$. This would require a Nyquist-sampling frequency of $10 \mathrm{GHz}$ in the traditional approach. We have chosen $B=20$ frequency bands equally spaced over the spectrum because

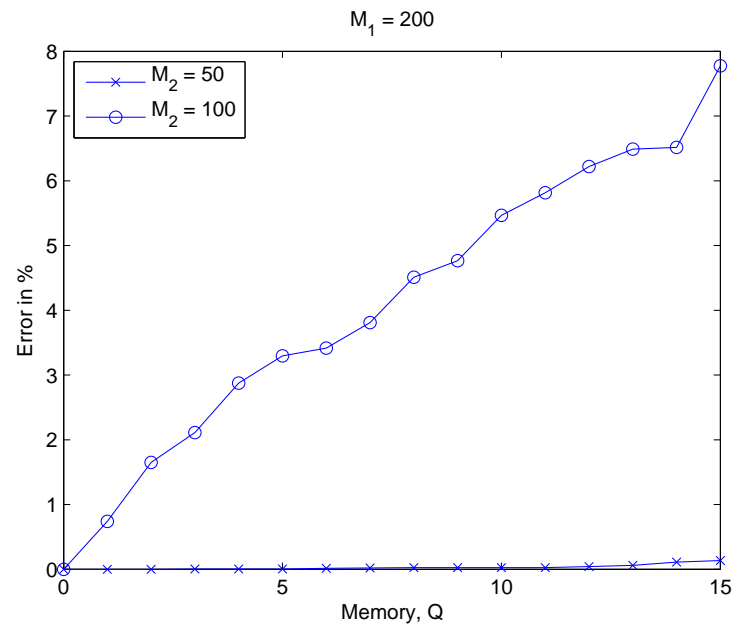

Fig. 1. Errors with different memory. $Q=0$ corresponds to taking full CS sample at each step

it is a reasonable range to show in the plots. We need about $N=400$ to get a good resolution of this spectrum. Because of the Gibbs phenomenon and the smoothing matrix $\mathcal{W}$, we get a sparsity in the edge spectrum $K \approx\left\|\mathbf{z}_{x}\right\|_{0} \approx 77$. From (1) we get $M=200$ which gives that we instead of a required $10 \mathrm{GHz}$ sampling rate can do it at $\frac{200}{400} f_{s}=5 \mathrm{GHz}$. This improvement is rather modest due to the relatively small $N$. In a real-world scenario, however, it is likely that $N$ is chosen as a larger value because of the need of higher spectrumresolution and since $M$ only grows logarithmically in $N$, potential gains are much larger.

As argued before, to get a good estimation of $\hat{\mathbf{R}}_{y}$, we need a sufficiently large $L$. By testing we find that $L=10000$ is required.

If the first sample needs $M_{1}=200$, the samples using memory need $M_{2}=C M_{1}$. Since $C=1 / 3, M_{2}=\frac{1}{3} 200=67$. This gives a further reduction in the sampling frequency $f_{s}=\frac{67}{200}<2 \mathrm{GHz}$.

In this paper we define an error to occur when the algorithm detects a frequency band as occupied when it is vacant or vice versa. In Fig. 1, we see the memory $Q$ plotted against the error (in percent), averaged from 1000 runs. We have chosen to plot the curves for $M_{2}=50<67$ (which is insufficient for our reconstruction) and $M_{2}=100$ (which should be sufficient for full recovery). In the following section we notice that indeed, considerable savings are obtained while maintaining full or nearly full performance.

\subsection{Analysis of the simulation results}

By examining Fig. 1, we notice that by taking the full $M=200$ at each sample, i.e. setting the memory $Q=0$, we get no errors at all. By applying the proposed algorithm with memory, we see that a reduction of $M_{2}$ down to 100 is possible without sacrificing any detection performance. We also notice that by choosing $M_{2}=$ 50 , which is insufficient for full recovery, errors occurs. The errors increase with the memory factor $Q$, as expected since an error in the detection is based on the total energy in each frequency band and, hence, is likely to remain in each step until a new full spectrum, sampled without utilizing old memory is estimated. This is true for both $M_{2}=50$ and $M_{2}=100$, but it shows clearer in $M_{2}=50$.

Fig. 2 shows how the reduction fraction varies with the memory assumed in our model. As one would expect, higher memory orders 


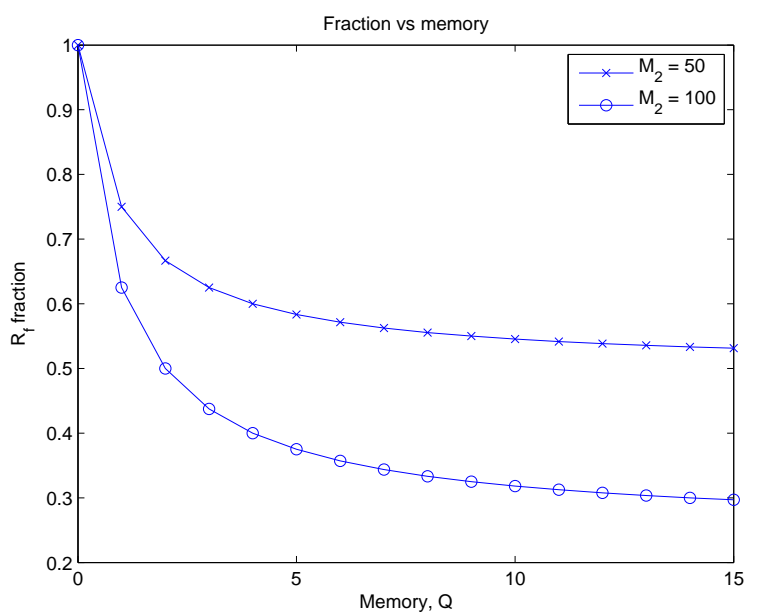

Fig. 2. The sample fraction gain at different memory. At $Q=0$ we take full CS sample at each iteration, i.e. no sampling improvement due to memory.

allow for larger reduction in terms of samples and frequency rate. This corresponds to the first equality in (17). The second equality in the same equation is achieved by setting $M_{2}=\frac{1}{3} M_{1}$ which would give a curve between the two curves plotted in the figure. Notice that the lower curve gives rise to errors in the process and the upper curve is close to error free.

In Fig. 3 we have represented the PSD estimated from $\mathbf{x}$ (top) and the corresponding reconstruction based on CS samples (bottom). Gibbs phenomenon is readily observed in the upper figure. With a larger number of taps in the filter, the step response would be faster, resulting in a sparser edge spectrum, as also discussed in section 4.2. If we compare the original signal with the reconstruction we observe a slight shift to the left in the latter with respect to the former. This shift is a natural effect of the implementation of the smoothing matrix $\mathcal{W}$. The detector should be aware of this shift and compensate for it appropriately. The rough outline of the reconstructed spectrum is mainly due to the estimation of the correlation matrix, rather than the CS process. However, since we are interested in detecting if there is a transmission in a certain frequency band this poses no practical problem.

Finally we remark on the trade-off in our memory based method. Larger memory allows for reduced frequencies but may impose errors in the detection. Also, as the memory grows, the robustness to sudden unexpected significant changes in the spectrum clearly decreases.

\section{CONCLUSION}

We have presented a method for estimating the power spectrum of a signal from CS samples intended for users with limited sampling capability, i.e. CR. The novelty of our method is that it does not rely on WSS assumptions of the CS sampled signal. Compared to traditional sampling at Nyquist rate, our suggested method may require more total samples for accurate estimation. However, these samples can be taken at a significantly lower rate than the Nyquist rate.

Our method extends to the case of slowly time-varying PSD. Further reductions is then possible in sampling rate and total number of samples are possible by employing an estimation technique based
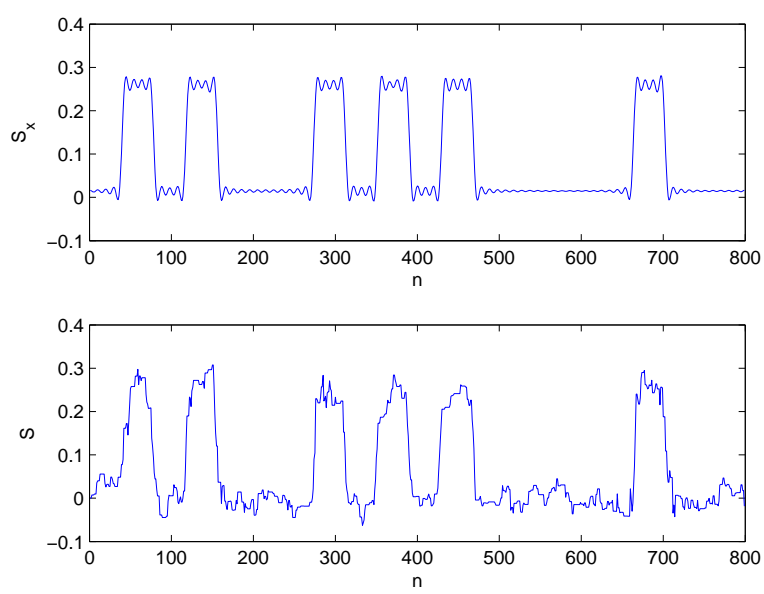

Fig. 3. top: PSD estimated from full Nyquist sampling, bottom: a typical CS-reconstruction from $\mathrm{M}=200$ samples.

on memory. Our simulation results show that the proposed memory based PSD estimation method is a promising alternative in scenarios where full-rate Nyquist sampling is not possible.

\section{Acknowledgements}

The authors would like to thank Dr. Michael Grant and Professor Stephen Boyd, Stanford University, for making the cvx toolkit for solving convex problems available to the public. Furthermore the authors would like to thank Professor Peter Händel, Royal Institute of Technology, for fruitful discussions regarding signal processing and Ricardo Blasco Serrano for his helpful comments.

\section{REFERENCES}

[1] D.L. Donoho, "Compressed sensing," IEEE Trans. Inf. Theory,, vol. 52, no. 4, pp. 1289 -1306, April 2006.

[2] Zhi Tian and G.B. Giannakis, "Compressed sensing for wideband cognitive radios," in IEEE Int. Conf. Acoustics, Speech and Signal Proc., 2007. ICASSP 2007., 15-20 2007, vol. 4, pp. IV-1357 -IV-1360.

[3] Y.L. Polo, Ying Wang, A. Pandharipande, and G. Leus, "Compressive wide-band spectrum sensing," in IEEE Int. Conf. Acoustics, Speech and Signal Proc., 2009. ICASSP 2009., 1924 2009, pp. $2337-2340$.

[4] Joseph Mitola, Cognitive Radio: An Integrated Agent Architecture for Software Defined Radio, Ph.D. thesis, Royal Institute of Technology (KTH), Stockholm, May 2000.

[5] Ying Wang, A. Pandharipande, Y.L. Polo, and G. Leus, "Distributed compressive wide-band spectrum sensing," Inf. Theory and Applications Workshop, pp. 178 -183, Feb 2009.

[6] S. Kirolos, T. Ragheb, J. Laska, M.E. Duarte, Y. Massoud, and R.G. Baraniuk, "Practical issues in implementing analog-toinformation converters," in The 6th Int. Workshop System-onChip for Real-Time Applications, Dec. 2006, pp. 141 -146. 
[7] Jason Laska, Sami Kirolos, Yehia Massoud, Richard Baraniuk, Anna Gilbert, Mark Iwen, and Martin Strauss, "Random sampling for analog-to-information conversion of wideband signals," in IEEE Dallas/CAS Workshop Design, Applications, Integration and Software, Oct. 2006, pp. 119-122.

[8] E.J. Candes and T. Tao, "Near-optimal signal recovery from random projections: Universal encoding strategies?," IEEE Trans. Inf. Theory, vol. 52, no. 12, pp. 5406-5425, Dec 2006.

[9] J.A. Tropp, J.N. Laska, M.F. Duarte, J.K. Romberg, and R.G. Baraniuk, "Beyond nyquist: Efficient sampling of sparse bandlimited signals," IEEE Trans. Inf. Theory,, vol. 56, no. 1, pp. 520 -544, Jan 2010.

[10] J.A. Tropp, M.B. Wakin, M.F. Duarte, D. Baron, and R.G. Baraniuk, "Random filters for compressive sampling and reconstruction," Acoustics, Speech and Signal Proc., 2006. ICASSP 2006 Proceedings. 2006 IEEE Int. Conf. on, vol. 3, pp. III -III, May 2006.

[11] Waheed U. Bajwa, Jarvis D. Haupt, Gil M. Raz, Stephen J. Wright, and Robert D. Nowak, "Toeplitz-structured compressed sensing matrices," Statistical Signal Proc., 2007. SSP '07. IEEE/SP 14th Workshop on, pp. 294 -298, Aug 2007.

[12] J. Romberg, "Sensing by random convolution," Computational Advances in Multi-Sensor Adaptive Processing, 2007.
CAMPSAP 2007. 2nd IEEE Int. Workshop on, pp. $137-140$, Dec 2007.

[13] E.J. Candes and T. Tao, "Decoding by linear programming," IEEE Trans. Inf. Theory, vol. 51, no. 12, pp. 4203 - 4215, Dec. 2005.

[14] E. J. Candes and J. Romberg, "Sparsity and incoherence in compressive sampling," Inverse Problems, vol. 23, no. 3, pp. 969-985, Jun. 2007.

[15] Scott Shaobing Chen, David L. Donoho, Michael, and A. Saunders, "Atomic decomposition by basis pursuit," SIAM Journal Scientific Computing, vol. 20, pp. 33-61, 1998.

[16] Chinh La and M.N. Do, "Tree-based orthogonal matching pursuit algorithm for signal reconstruction," Image Processing, 2006 IEEE Int. Conf. on, pp. 1277 -1280, Oct 2006.

[17] M. Grant and S. Boyd, "CVX: Matlab software for disciplined convex programming, version 1.21," http://cvxr.com/cvx, Jul. 2010.

[18] H. L. Van Trees, Detection, Estimation, and Modulation Theory, Part I, Wiley-Interscience, 1 edition, Sep. 2001.

[19] P. Stoica and R. Moses, Spectral Analysis of Signals, Pearson Prentice Hall, 2005. 